\author{
Abstracta Iranica \\ Abstracta Iranica Revue bibliographique pour le domaine irano-aryen \\ Volume 37-38-39 | 2018 \\ Comptes rendus des publications de 2014-2016
}

\title{
Ali Mozaffari (ed.). World Heritage in Iran. Perspectives on Pasargadae
}

\section{Sébastien Gondet}

\section{(2) OpenEdition \\ 12 Journals}

\section{Édition électronique}

URL : http://journals.openedition.org/abstractairanica/45865

DOI : 10.4000/abstractairanica.45865

ISBN : 1961-960X

ISSN : 1961-960X

Éditeur :

CNRS (UMR 7528 Mondes iraniens et indiens), Éditions de l'IFRI

Référence électronique

Sébastien Gondet, « Ali Mozaffari (ed.). World Heritage in Iran. Perspectives on Pasargadae», Abstracta Iranica [En ligne], Volume 37-38-39 | 2018, document 5, mis en ligne le 30 décembre 2018, consulté le 28 septembre 2020. URL : http://journals.openedition.org/abstractairanica/45865 ; DOI : https:// doi.org/10.4000/abstractairanica.45865

Ce document a été généré automatiquement le 28 septembre 2020

Tous droits réservés 


\title{
Ali Mozaffari (ed.). World Heritage in Iran. Perspectives on Pasargadae
}

\author{
Sébastien Gondet
}

\section{RÉFÉRENCE}

Ali Mozaffari (ed.). World Heritage in Iran. Perspectives on Pasargadae. Farnham: Ashgate Publishing Limited, 2014, xvi+262 p. (Heritage, Culture and Identity Series)

1 Alors que ces dernières décennies l'Iran poursuit une politique volontariste d'inscription de nombreux sites et paysages culturels sur la Liste du patrimoine mondial de l'Unesco, voire par exemple la récente inscription du «Paysage archéologique sassanide du Fars » en 2018, cet ouvrage propose une analyse croisée des enjeux et des conséquences d'une telle patrimonialisation sur le site de Pasargades, inscrit assez récemment en 2004. L'éditeur de ce livre, architecte de formation et s'intéressant aux études du patrimoine, a demandé à dix contributeurs d'horizons très divers, pour certaines parties prenantes de l'étude et de la mise en valeur du site, de donner leur propre éclairage sur le site. Il en résulte un ouvrage résolument pluridisciplinaire, ce qui en constitue tout l'intérêt, examinant la notion de patrimoine à Pasargades, et son devenir, à travers les yeux de l'anthropologie, de l'archéologie, de l'architecture, de l'économie, de la science politique, de la philosophie et de l'épistémologie.

2 Ali Mozaffari, "Conceptualising a World Heritage Site”, p. 1-28

3 Cet article constitue l'introduction de l'ouvrage. Il traite tout d'abord des évolutions, sur le temps long et depuis sa fondation à l'époque achéménide, de la perception de Pasargades par les populations locales, par les différents pouvoirs en place et par les scientifiques. Ces évolutions ont eu un impact profond sur les fonctions et la morphologie du site et en ont fait un des éléments majeurs du Patrimoine iranien. La seconde partie se propose d'illustrer ces dynamiques, parfois contradictoires, à partir de la situation actuelle. Ces dernières décennies, Pasargades, plus exactement la Tombe 
de Cyrus, est devenu un des points de fixation de conflits sur la (re)définition d'une identité collective nationale. Enfin, cet article se conclut sur une présentation des contributions rassemblées dans le volume et des grilles d'analyse qu'elles offrent sur le patrimoine de Pasargades.

Rémy Boucharlat, "Archaeological Approaches and their Future Directions in Pasargadae", p. 29-59

5 L'A. présente un historique des recherches archéologiques à Pasargades et dans sa région divisée en trois grandes périodes, chacune conduisant une redéfinition archéologique du site. Alors que l'identification du site à Pasargades avait été perdue, les savants-voyageurs du $19 \mathrm{e}$ s. vont peu à peu « redécouvrir » le site. Son association à l'antique capitale de Cyrus ne sera définitivement confirmée qu'avec les premières fouilles mises en œuvre à la fin des années 1920. Les fouilles plus systématiques des années 1950-1960 permettront ensuite de définir le site comme une capitale-jardin au plan très ouvert. Ce plan sera précisé et élargi lors de la troisième grande phase de recherches archéologiques, en grande partie pilotées par l'A. au cours de la première décennie des années 2000, en se concentrant sur l'utilisation de nouvelles méthodes de cartographie à large échelle. Parallèlement, les archéologues ne se sont plus seulement intéressés au seul site de Pasargades mais également à l'organisation de son territoire. En conclusion, l'A., s'appuyant sur son expérience personnelle, brosse les grandes lignes d'un programme de recherche qu'il resterait à développer sur et autour de Pasargades et démontre l'important potentiel du site et de son territoire, qui restent encore largement à définir archéologiquement.

(a) Talebian, "The Current State of Heritage Management at Pasargadae: Problems and Prospects", p. 61-89

Outre les arguments qui ont conduit à l'inscription de Pasargades sur la Liste de patrimoine mondial de l'Unesco, cet article détaille les conséquences qu'a eues le classement du site sur les politiques mises en œuvre pour assurer sa gestion et sa préservation. Au sein de l'Organisation iranienne du patrimoine culturel, de l'artisanat et du tourisme, l'A. était alors en charge, entre autres fonctions, de la constitution du dossier de classement du site. Il livre donc un témoignage de première main sur les coulisses d'une telle entreprise, depuis les réorganisations administratives nécessaires jusqu'aux difficultés rencontrées pour associer les populations locales à la préservation du paysage autour du site. En outre, le classement de Pasargades en 2004 a servi de point d'appui à de nouvelles dynamiques non seulement de mise en valeur mais également de recherches sur le site dont l'A. dresse les priorités.

William M. Taylor, "Archaeology and Useful Knowledge: Pasargadae and the Rationalities of Digging 'Real' Places”, p. 91-113

Cette contribution, à visée épistémologique, traite de l'usage qui est fait de l'archéologie pour légitimer certains discours politiques, la mise au jour de preuves matérielles leur apportant des preuves scientifiques irréfutables. L'archéologie présente pourtant de nombreux biais, de par ses méthodes et ses objectifs, qui nécessitent de questionner les découvertes archéologiques et surtout leurs interprétations par les archéologues. Dans un volume centré sur Pasargades, ce site ne sert pourtant que de toile de fond à un discours certes intéressant mais qui reste très général et théorique. Le thème des relations tumultueuses entre archéologie et 
politique à Pasargades est traité de manière plus détaillée dans plusieurs autres articles de ce volume.

Riccardo Baldissone, "The Costs of Paradise: Temporalisations of Place in Pasargadae", p. $115-138$

11 L'aménagement d'un jardin, d'un " paradis ", à Pasargades et surtout la construction de la tombe de Cyrus à l'intérieur de cet espace, qui constitue en fait le principal objet de réflexion de cet article, permet à l'A. de développer une discussion sur les évolutions de la symbolique du lieu à partir de l'époque achéménide jusqu'aux époques modernes. D'abord symbole de l'emprise du Grand Roi sur l'espace, la tombe monumentale est peu à peu associée à la figure mythique de la Mère de Salomon au cours des périodes médiévales. Aux époques modernes, on cherchera à réattribuer exclusivement la tombe à Cyrus et au glorieux passé achéménide en tentant d'effacer tous les ajouts islamiques. L'A. plaide pour qu'un compromis soit trouvé afin de concilier ces différents récits symboliques.

12 Soheila Shahshahani, "Pasargadae, Mādar Soleimān and Leilā: Placing Pasargadae within its Present Rural Context", p. 139-154

Cet article rend compte d'une étude ethnographique du village de Mādar Soleimān qui jouxte le site de Pasargades. Elle s'appuie en grande partie sur des entrevues réalisées par l'A. avec la cheffe du conseil du village. Tout en retraçant l'histoire du développement du village, les informations contenues ici ayant par ailleurs mérité d'être mieux sourcées, l'A. démontre comment les populations locales ont pris soin de la tombe de Cyrus, devenu lieu de pèlerinage, au cours des derniers siècles. Toutefois, l'inscription du site à l'Unesco a introduit l'administration du patrimoine iranien comme acteur principal de la protection du site. Les habitants ont dès lors eu à gérer de nombreux conflits avec cette administration dont les décisions freinent parfois les projets de développement du village.

Tod Jones, Mohammad-Hassan Talebian, "Perspectives and Prospects for Cultural Tourism in the Pasargadae Region", p. 155-172

Cet article traite du site de Pasargades d'un point de vue économique et de son potentiel pour développer le tourisme dans la région. Une première partie expose les données récentes concernant le nombre de visiteurs sur le site ainsi que leur profil et leurs motivations. Elles sont replacées dans le contexte plus large des politiques de développement du tourisme en Iran au cours des dernières décennies. Une seconde partie traite des différents facteurs permettant un développement durable du tourisme autour des sites patrimoniaux, tels que définis par des experts internationaux, puis les explorent à l'échelle de Pasargades et de sa région. Plusieurs pistes sont avancées pour améliorer, dans le futur, l'expérience du site par les visiteurs.

16 Jennifer Harris, "The Past in the Present: Using Poetics as an Interpretative Strategy at Pasargadae", p. 173-195

17 Se basant sur une réflexion théorique concernant la notion de "paysage culturel ", mise en avant par l'Organisation du patrimoine iranien dans le dossier de classement de Pasargades, l'A. plaide pour mettre en avant les expériences sensorielles et poétiques que procurent la visite du site. Le paysage de Pasargades possède en effet un fort potentiel pour laisser libre cours à l'imagination du visiteur sans lui imposer une vision du site figée par sa patrimonialisation. Une telle stratégie de mise en valeur permettrait en outre d'apaiser les conflits existants autour de l'interprétation 
historique des vestiges et des tentatives d'appropriation par les différentes parties prenantes.

Ali Mozaffari, Nigel Westbrook, "The (Unfinished) Museum at Pasargadae", p. 197-224

Lors des restructurations qu'a connues le site au cours des années 1970, dans le cadre des célébrations du Jubilé de 1971, la construction d'un musée à Pasargades avait été initiée à quelques centaines de mètres de la Tombe de Cyrus. Sa construction s'est arrêtée en 1979 au moment de la Révolution. Il est depuis resté à l'état de "ruine " avant que les travaux ne reprennent en 2011. Les A. retracent l'histoire de ce musée, conçu par l'architecte, Hossein Amanat, et offrent une réflexion sur sa conception. Cet exposé s'appuie en grande partie sur une interview de l'architecte iranien alors en charge du projet. Il apparaît que le plan du musée illustre avant tout les grands courants architecturaux de l'époque qui se sont largement diffusés dans les écoles d'architecture iraniennes.

Elham Shamoradi, Ebrahim Abdollahzadeh (Trad. par Ali Mozaffari), "Antinomies of Development: Heritage, Media and the Sivand Dam Controversy”, p. 225-253

La construction du barrage de Sivand, initié au début des années 1990 dans la vallée du Tang-e Bolaghi voisine de Pasargades, a fait l'objet, au milieu des années 2000, de vives controverses à propos des dangers que sa mise en eau ferait courir au patrimoine. Les AA. exposent les différentes parties prenantes, leur nature, leurs objectifs et leur stratégie de communication, depuis les propriétaires terriens, les groupes d'activistes en Iran et extérieurs, les médias ainsi que les acteurs institutionnels. Les menaces, supposées, sur le patrimoine ont servi de support à un discours politique de délégitimisation d'un pouvoir en place et d'une administration du patrimoine qui auraient été peu soucieux de préserver le grand site de Pasargades. Ce discours, basé sur des fausses nouvelles et se nourrissant des erreurs de communication publique, s'est largement diffusé sur internet et dans certains médias plus classiques, basés hors d'Iran suivant un processus analysé dans le détail par les AA.

\section{AUTEURS}

\section{SÉBASTIEN GONDET}

UMR 5133 CNRS-Université de Lyon 